\title{
Cenários de avaliação das restrições à mobilidade dos pedestres em espaços públicos: o caso de um campus universitário
}

\author{
Fabíola de Oliveira Aguiar'; Rui António Rodrigues Ramos²; \\ Antônio Nélson Rodrigues da Silva ${ }^{3}$
}

\begin{abstract}
Resumo: Este trabalho apresenta uma avaliação das condições de mobilidade do pedestre de acordo com o nível disponível de acessibilidade das calçadas para cada tipo de usuário observado. Através do método de avaliação multicritério em ambiente de Sistemas de Informação Geográfica, é apresentado um estudo de caso em um campus universitário para os seguintes grupos de usuários: 1) sem deficiência aparente, 2) cadeirantes, 3) com deficiência visual e 4) com restrição de locomoção. Os resultados identificaram locais com diferentes níveis de acessibilidade para cada tipo de usuário, evidenciando que a disposição das edificações dentro do campus e o grau de importância dessas edificações atribuído pelos usuários geram bolsões com níveis de acessibilidade mais elevados. As conclusões sugerem que o método é adequado e promissor para identificar espaços urbanos com soluções ineficientes para a mobilidade de grupos específicos da população usuária.
\end{abstract}

\begin{abstract}
This paper presents an assessment of the pedestrians' mobility conditions according to the level of accessibility of the pathways available for each type of user observed. Through a multicriteria evaluation carried out in a Geographic Information System, a case study was developed in a university campus for the following groups of users: 1) without apparent disabilities, 2) wheelchair users, 3) with visual disabilities, and 4) with mobility constraints. The results identified areas with different accessibility levels for each user type. They show that the arrangement of buildings within the campus and the degree of importance attributed by the users of these buildings create spots with higher levels of accessibility. The findings suggest that the method is a suitable and promising alternative for identifying urban locations with ineffective solutions to the mobility of specific population groups.
\end{abstract}

\section{INTRODUÇÃO}

O termo mobilidade urbana diz respeito à facilidade de deslocamento de pessoas e bens dentro das cidades e vem sendo introduzido nos estudos de planejamento urbano e de transportes, entre outros enfoques, para ratificar a importância do tema da acessibilidade em meio urbano e em espaços de uso coletivo. Tendo em vista que muitas atividades hoje envolvem deslocamentos, a existência de barreiras impedindo esses deslocamentos pode limitar essas atividades. Esse fato sugere a necessidade de pesquisas que permitam avaliar o papel dos transportes como um fator de inclusão ou exclusão de indivíduos com restrições de mobilidade (Mohammadian e Bekhor, 2008; Schmöcker, 2009).

A mobilidade - que aqui será abordada apenas no que se refere aos deslocamentos físicos a pé - deve estar intrínseca às necessidades mais básicas de qualquer

\footnotetext{
${ }^{1}$ Fabíola de Oliveira Aguiar, Universidade Estadual do Maranhão Departamento de Arquitetura e Urbanismo, São Luís, MA, Brasil. (e-mail: fabiola agui@hotmail.com).

${ }^{2}$ Rui António Rodrigues Ramos, Universidade do Minho, Escola de Engenharia, Minho, Portugal. (e-mail: rui.ramos@civil.uminho.pt).

${ }^{3}$ Antônio Nélson Rodrigues da Silva, Universidade de São Paulo, Escola de Engenharia de São Carlos, São Carlos, SP, Brasil. (e-mail: anelson@sc.usp.br).
}

Manuscrito recebido em 7/12/2008 e aprovado para publicação em $1 / 6 / 2009$. Este artigo é parte de TRANSPORTES, volume XVII, número 2, dezembro de 2009. ISSN: 1415-7713. pessoa. Isso não pode excluir aquelas que, por algum motivo, possuam restrições ao efetuar os seus deslocamentos. Por conseguinte, alguns países têm direcionado ações e pesquisas para o tema, com a finalidade de garantir o deslocamento do maior número possível de pessoas, independentemente das suas condições de locomoção (como discutido em Schmöcker et al., 2008, e Páez et al., 2009). Muitas dessas iniciativas são desdobramentos do Programa de Ação Mundial para Pessoas com Deficiência da Organização das Nações Unidas, de 1983, e da Convenção de Direitos das Pessoas com Deficiência, documento assinado por mais de uma centena de países (ONU, 2007).

Do ponto de vista de legislação específica, o exemplo da ADA (Americans with Disabilities Act, ver detalhes em http://www.ada.gov/) é uma das iniciativas mais conhecidas, tendo sido aprovada em 1990 pelo Congresso dos Estados Unidos da América. Segundo Little (1995), os principais objetivos almejados com a ADA eram assegurar o acesso de indivíduos com dificuldades de locomoção a edifícios e ao transporte público. Desta forma, a iniciativa pretendia que esses indivíduos passassem a ter maiores oportunidades de emprego e de educação, bem como de acesso ao sistema de seguridade social. Isto demonstra como soluções para os espaços urbanos e de uso coletivo destinados à circulação de pedestres preconizadas por leis e normas têm sido, cada vez mais, orientadas para contemplar as necessidades das pessoas com dificuldade de locomoção. 
Em diversas situações referentes à facilidade de deslocamento, principalmente no que diz respeito ao modo a pé, os termos mobilidade e acessibilidade estão diretamente relacionados e muitas vezes se confundem. Isto pode ser explicado pelo fato de que quando se aumenta o nível de acessibilidade a determinado espaço, espera-se aumentar também as condições de mobilidade oferecidas aos usuários desse espaço. Isso foi discutido, entre outros, por Páez et al. (2009), cujo trabalho se constitui em uma referência útil para intervenções potenciais para melhorar as condições de acessibilidade. Entre as intervenções potenciais estão: a interferência em fatores que afetam a mobilidade, tais como as condições econômicas, e alterações na distribuição espacial das oportunidades, de forma a promover a aproximação entre indivíduos e oportunidades.

Assim, para o modo a pé, pode-se afirmar que a acessibilidade está associada às oportunidades disponibilizadas pelo espaço urbano. Por outro lado, a mobilidade está ligada à condição de um indivíduo se deslocar, mas esta condição depende da performance do espaço (níveis de acessibilidade) e das características do próprio indivíduo (capacidade de locomoção). Como enfatizado por Church e Marston (2003), ações voltadas a integrar e a dar qualidade à circulação em espaços urbanos são essenciais para garantir níveis adequados de acessibilidade e assim promover melhores condições de mobilidade.

A maioria dos estudos de planejamento urbano e de transporte não considera, na prática, que os pedestres tenham características distintas na sua capacidade de locomoção, o que por vezes os torna mais vulneráveis às soluções preconizadas. Pode-se considerar que a capacidade de locomoção de cada indivíduo é influenciada por diversos fatores: a idade (penalizando as crianças e os idosos), a condição física permanente (penalizando pessoas com deficiência física, sensorial ou mental; pessoas com pequena ou grande estatura etc.) ou provisória (tais como gestantes a partir do sexto mês, obesos etc.) e, até mesmo, o estado momentâneo (penalizando pessoas que empurram carrinhos, carregam objetos de grande peso ou volume etc.).

O objetivo do presente trabalho consiste em investigar o nível de acessibilidade proporcionado por calçadas e travessias destinadas ao pedestre (incluindo pessoas com dificuldade de locomoção - PDLs) e a sua relação com fatores que possam condicionar a mobilidade desse pedestre. Para tanto, pretende-se identificar as deficiências e/ou ineficiências dos espaços de circulação urbana destinados ao pedestre através de um método de avaliação que forneça parâmetros para mapear as condições de mobilidade e servir de suporte à análise no apoio à decisão para planejadores e administradores urbanos. Para tal, foi proposto um estudo de caso que permitiu avaliar o nível de acessibilidade em calçadas e travessias no campus de Gualtar da Universidade do Minho, em Braga, Portugal. O estudo avalia as condições de mobilidade oferecidas a quatro grupos de usuários com características distintas. A análise foi realizada por meio da aplicação do Método de Avaliação Multicritério, com implementação do modelo em ambiente de Sistema de Informação Geográfica (SIG).

\section{CONTEXTUALIZAÇÃO}

Segundo Feijó (2002), a concretização do direito de ir e vir é fundamental para a PDL, pois a habilita para usufruir muitos outros (por exemplo, o direito ao trabalho, lazer, escola etc.). Por isto, a mobilidade tem sido tema de grande relevância em nível mundial, amparado pelos princípios do "Desenho Universal", principalmente no caso da mobilidade de pedestres. Prado (1997) cita que tais princípios preconizam que as cidades devem ser acessíveis a qualquer pessoa, desde seu nascimento até sua velhice.

Os dados referentes a Portugal, obtidos do Censo 2001, sinalizavam para a existência de aproximadamente seiscentas mil pessoas com deficiência (PCDs), o que representava uma taxa de $6,1 \%$ da população total (Portugal, 2006 e INE, 2002 apud Almeida, 2006). Dentro deste valor, a taxa de incidência de pessoas com deficiência visual era a mais elevada, representando $1,6 \%$ do total da população. Os indivíduos com deficiência auditiva registravam uma percentagem mais baixa $(0,8 \%)$. Com relação à deficiência motora, esta representava $1,5 \%$ da população total. A taxa de pessoas com deficiência mental representava $0,7 \%$. A paralisia cerebral foi o tipo de deficiência com a menor incidência na população recenseada $(0,1 \%)$. O conjunto das outras deficiências, que inclui as não consideradas em qualquer dos outros tipos, situou-se em $1,4 \%$ do total de indivíduos. Se forem somadas às PCDs as pessoas com restrição de movimento (PRMs - como idosos, crianças, obesos, gestantes após o sexto mês, indivíduos com visão subnormal) e as pessoas com deficiência temporária, esse número aumenta consideravelmente. Os dados do Censo 2001 revelavam ainda que, em Portugal, a população de idosos (pessoas com 65 anos ou mais) representava 16,4\% da população total (INE, 2002).

Diante do exposto, fica evidente que o número de PCDs e PRMs é significativo entre o universo de pedestres, pelo que é necessário criar condições apropriadas para a sua mobilidade. Todavia, os espaços públicos urbanos possuem frequentemente diversas situações que constituem verdadeiros impedimentos à sua mobilidade, tais como: existência de degraus, rampas muito inclinadas, mobiliário urbano mal posicionado e 
veículos estacionados nos espaços destinados à circulação dos pedestres.

A exemplo de outros países, Portugal pretende também reverter alguns dos problemas identificados a partir de um aparato legal. Assim, segundo Martins (2008) a PCD é tratada na Constituição da República Portuguesa, especificamente com relação ao tema aqui exposto, segundo competências, a saber: é dever do Estado a promoção do bem-estar e qualidade de vida da população e a igualdade real e jurídico-formal entre todos os portugueses (alínea $d$ ) do artigo $9^{\circ}$ e artigo $1^{\circ}$ ]. A alínea $d$ ) do artigo $3^{\circ}$ da Lei de Bases da Prevenção, Habilitação, Reabilitação e Participação das Pessoas com Deficiência (Lei n 38/2004, de 18 de agosto) determina a promoção de uma sociedade para todos através da eliminação de barreiras e da adoção de medidas que visem a plena participação da pessoa com deficiência. A matéria sobre acessibilidade foi objeto de regulação normativa através do Decreto-Lei $n^{\circ}$ 123/1997, de 22 de maio, que introduziu normas técnicas visando à eliminação de barreiras urbanísticas e arquitetônicas nos edifícios públicos, equipamentos coletivos e vias públicas. Mais recentemente estas normas foram atualizadas e procedeu-se à introdução de novas normas técnicas aplicáveis em âmbito mais amplo. Assim, o Decreto-Lei n. ${ }^{\circ}$ 163/2006, de 8 de agosto, que trata da aprovação do regime da acessibilidade aos edifícios e estabelecimentos que recebem público, via pública e edifícios habitacionais, revogou o Decreto-Lei n. ${ }^{\circ}$ 123/1997.

Também outros dispositivos legais, como as Posturas Municipais 141/81 e 142/81 criadas pela Câmara Municipal de Lisboa, que definiram normas e conceitos de acessibilidade e eliminação de barreiras arquitetônicas, ainda subsistem como instrumentos auxiliares de trabalho de projetistas e interventores do espaço público (Martins, 2008). O conjunto de normas que contém o RAMP - Regulamento Municipal para Promoção da Acessibilidade e Mobilidade Pedonal (Edital n 29/2004, de 06 de julho) estabelece parâmetros atualizados de acessibilidade física para Lisboa. De modo geral, as ações ainda se limitam a propor a readequação de espaços públicos sem o conhecimento das verdadeiras características físicas de locomoção das PCDs e PRMs. Sob esta ótica, o RAMP cita que a cidade existente exclui uns, exige demasiado esforço a outros e é um fator de desconforto e insegurança para a maioria. Deste modo, ainda não corresponde às verdadeiras necessidades da sua população do ponto de vista físico, informativo e comunicacional.

No Brasil, Duarte e Cohen (2004) e Cohen (2006) reportam ser comum que a construção de rampas nas esquinas seja considerada "suficiente" para taxar o projeto urbano de "projeto inclusivo". As mesmas autoras citam ainda que os aspectos referentes ao espaço, como a distância (perto e longe) e a forma do percurso, devem ser medidas pelo esforço e pelo cansaço da PDL e não podem ou não devem ser compreendidos a partir de referenciais de pessoas que não apresentam dificuldade em sua locomoção. Portanto, o "tempo de percurso" é um fator ou critério determinante para avaliar as condições de mobilidade. Contudo, além do tempo de percurso, outro critério relevante para análise da mobilidade dos usuários é a importância relativa dos vários destinos-chave para os usuários, como refere Mendes (2000). Assim, esse aspecto que tem relação direta com o nível de acessibilidade também foi integrado no modelo de análise desenvolvido neste trabalho. Pois, para a realização de uma viagem, seja por qualquer modo de transporte (neste caso foram consideradas apenas as realizadas pelo modo a pé) os propósitos dessa viagem estão relacionados aos destinos. Estes, por sua vez, podem possuir importâncias diferentes (pesos) para o usuário, de acordo com a sua utilização. Importa ressaltar que a idéia de acessibilidade está mais intimamente relacionada à capacidade de alcançar destinos desejados e/ou necessários do que propriamente ao movimento stricto sensu.

De acordo com Rodrigues da Silva et al. (2008) processos de análise que utilizam diversos critérios para serem avaliados e combinados podem ser realizados segundo uma avaliação multicritério, que permite a obtenção de índices. Os mesmos autores citam que a integração dos modelos em ambiente SIG simplifica a análise, uma vez que o mesmo tem capacidade de trabalhar grande quantidade de informação sobre o território. Tal procedimento permite facilidade na tomada de decisão através da visualização dos resultados obtidos em vários cenários de avaliação, como será aqui demonstrado.

\section{METODOLOGIA}

Para a realização deste trabalho adotou-se como princípio que as condições de mobilidade dos pedestres podem ser medidas pelo nível de acessibilidade dos espaços. A acessibilidade, por sua vez, pode ser medida segundo a facilidade com que os diferentes usuários efetuam os percursos para vários destinos-chave. Assim, para o sítio em que foi desenvolvido o estudo de caso foi necessário: construir uma rede para identificar menores caminhos, identificar os destinos-chave e seus respectivos pesos, determinar velocidades e tempos para cada grupo de usuários, determinar impedâncias nos trechos e aplicar o modelo para obtenção dos índices de acessibilidade, como detalhado nos itens a seguir. 


\subsection{Construção da rede e identificação dos destinos-chave com seus respectivos pesos}

Numa primeira fase foi necessário efetuar o reconhecimento da área através de levantamento de campo, o que envolveu análises técnicas e estudos de observação. Desta forma foi possível identificar e caracterizar os trajetos efetuados pelos pedestres, medindo-se nos diversos trechos as respectivas inclinações transversais e longitudinais, larguras mínimas efetivas, tipo e estado do piso, existência de degraus e obstáculos. A Figura 1 ilustra uma das etapas do levantamento de campo com a aferição das inclinações das calçadas. Esse levantamento permitiu elaborar a rede em ambiente SIG-T (neste caso, através do software Trans$C A D)$.

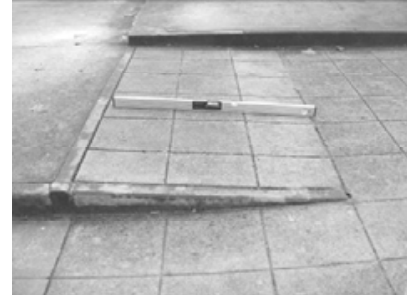

(a)

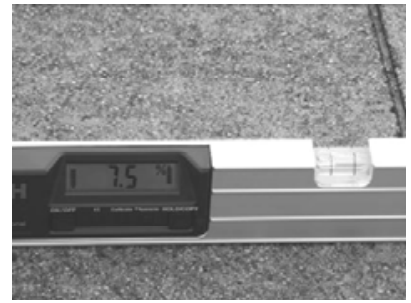

(b)
Figura 1. Exemplo de aferição da inclinação de uma calçada com inclinômetro digital (a) e detalhe do valor aferido em porcentagem (b)

Foram utilizados os valores dos pesos decorrentes da importância atribuída pelos usuários aos prédios (destinos-chave) de acordo com sua utilização, com base nos resultados da pesquisa de opinião realizada por Rodrigues (2001). Naquela altura, foram aplicados questionários aos usuários do campus - alunos, docentes e funcionários. Ainda de acordo com a mesma pesquisa de opinião foram extraídos os valores dos percursos máximos, em termos de distância, que os usuários estariam dispostos a fazer para alcançar cada um destes destinos. O dado de distância máxima per- mitiu obter, posteriormente, o tempo máximo de percurso aceitável por grupo de usuários.

\subsection{Determinação de velocidade e tempo para cada grupo de usuários}

As velocidades utilizadas para os quatro grupos de pedestres considerados foram as mesmas obtidas e utilizadas em Aguiar et al. (2008). Logo, para efeito de aplicação do método, as velocidades adotadas em plano horizontal foram: $1,38 \mathrm{~m} / \mathrm{s}$ (cerca de $5 \mathrm{~km} / \mathrm{h}$ ) para pessoas sem deficiência aparente; $1,35 \mathrm{~m} / \mathrm{s}$ para cadeirantes; $1,19 \mathrm{~m} / \mathrm{s}$ para pessoas com restrição de mobilidade (idosos) e $0,99 \mathrm{~m} / \mathrm{s}$ para pessoas com deficiência visual (cego). No caso de planos inclinados, escadarias etc., foram consideradas impedâncias em função da alteração das condições de locomoção relativamente ao plano horizontal, o que influenciou os valores de velocidade de cada grupo (ver item 3.3). O tempo de percurso derivou das diferentes velocidades dos usuários e das distâncias reais de cada percurso medidas na rede elaborada. Entretanto, devido à existência de obstáculos, este tempo foi condicionado à velocidade adotada quando da existência de alguma impedância.

\subsection{Determinação das impedâncias nos trechos}

O tempo de percurso foi condicionado pelas impedâncias resultantes das características físicas dos espaços avaliados, de acordo com as restrições à locomoção de cada grupo de usuários. Como exemplo de impedância pode ser citada a existência de escadarias ou calçadas sem rebaixamento. Para um usuário sem deficiência aparente, a escadaria pode representar um pequeno acréscimo de esforço. Já para um cadeirante representa uma barreira intransponível, o que se traduz numa velocidade nula (ele não pode transpor esse trecho e tem que buscar uma rota alternativa, certamente mais longa e demorada). A Figura 2 mostra uma parte da rede de pedestres utilizada e a análise realizada sobre o

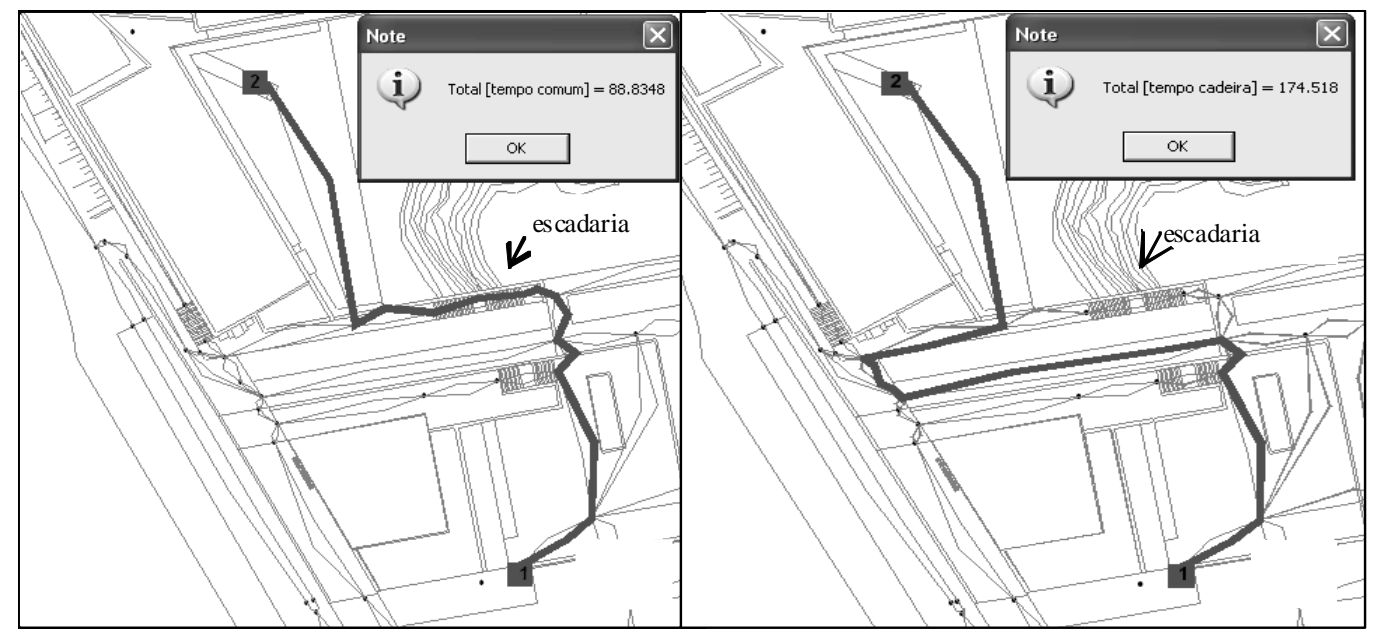

(a)

(b)

Figura 2: Tempo despendido por pessoas sem deficiência aparente (a) e por pessoas com deficiência física - cadeirantes (b) quando existe uma impedância significativa 
menor caminho em função do tempo despendido por pessoas sem deficiência aparente e com deficiência física (cadeirante). Considerando que ambas partem da mesma origem e chegam ao mesmo destino, o tempo do cadeirante é quase duas vezes maior pelo fato de existir uma escadaria. A alternativa, para ele, é contornar essa barreira optando por um percurso que se evidencia mais longo (esse aspecto foi também bastante discutido por Church e Marston, 2003).

\subsection{Aplicação do modelo para obtenção dos índices de acessibilidade}

Os procedimentos de combinação de critérios utilizados nesta pesquisa para análise no processo de decisão foram: a Combinação Linear Ponderada (WLC, derivado do inglês Weighted Linear Combination) e a Média Ponderada Ordenada (OWA, do inglês Ordered Weighted Average). A avaliação através do procedimento WLC, que combina os critérios segundo uma média ponderada, permite uma compensação das qualidades entre os critérios. O procedimento OWA, além de combinar os critérios através de uma média ponderada (WLC), ainda aplica pesos aos critérios ordenados, resultando em diferentes cenários. Estes cenários, elaborados a partir de combinações dentro de um espaço estratégico de tomada de decisão, são definidos por pontos de decisão (ou controle). O espaço estratégico de decisão é aproximadamente triangular, definido por um lado pela atitude de risco e, por outro lado, pelo nível de compensação (trade-off), como se observa na Figura 3.

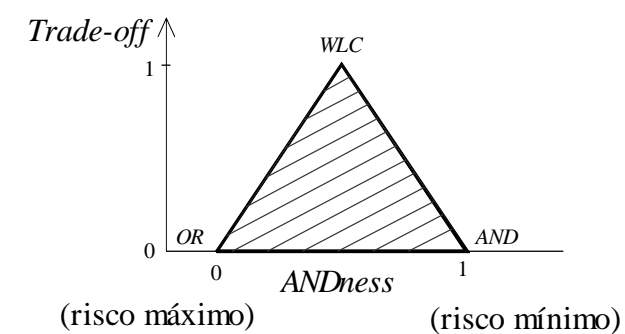

Figura 3. Espaço estratégico de decisão para o procedimento OWA

A média dos tempos máximos (Tm), para cada destino-chave, foi calculada de acordo com a média das distâncias máximas indicadas pelos usuários na pesquisa de opinião realizada por Rodrigues (2001). Os tempos reais $(\mathrm{Tr})$ foram extraídos da matriz de menores caminhos em função do menor tempo. Com base nestes dois valores (Tm e Tr), foi possível obter o tempo normalizado (Tn) para cada grupo de usuários. O objetivo é, para todos os destinos-chave, transformar qualquer escala de avaliação em uma escala comparável, onde os seus valores se enquadrem num intervalo normalizado. Neste caso, os resultados expressam o grau de pertinência a um intervalo de valores entre zero e um, delineando uma variação contínua desde a não-pertinência (acessibilidade nula) até à completa pertinência (acessibilidade máxima). Os pontos de controle das curvas fuzzy constituem pontos críticos que, consoante os casos, deverão ser escolhidos tendo em conta o seu significado. Neste caso, a normalização foi efetuada adotando-se uma função linear para cada destino-chave pelo processo de fuzzification (Rodrigues da Silva et al., 2008). Portanto, a função fuzzy aplicada ao custo de viagem que foi considerada nesta pesquisa é relativa ao tempo.

Os pesos dos destinos-chave utilizados também foram obtidos da pesquisa de opinião realizada por Rodrigues (2001), como citado no item 3.1. Desta forma, e de acordo com Mendes (2000), o índice de acessibilidade de todos os locais do campus em relação aos destinos-chave analisados pode ser obtido através da Equação 1.

$$
A_{i}^{u}=\sum f^{u}\left(c_{i j}\right) \cdot w_{j}
$$

em que,

$A_{i}^{u}$ : índice de acessibilidade de um local $i$ para o grupo de usuários $u$;

$f^{u}\left(c_{i j}\right)$ : função linear tempo-custo para o grupo de usuários $u$ entre o local $i$ e o destino-chave $j$ (função do Tn);

$w_{j}$ : peso do destino-chave $j$.

O cálculo do índice de acessibilidade deve ser efetuado para cada grupo de usuários " $u$ ", uma vez que o tempo de percurso para cada grupo é considerado diferente. Assim, a Equação 1 foi aplicada de forma a identificar os níveis de acessibilidade que cada grupo possui. Obtidos os valores dos índices de acessibilidade $A_{i}^{u}$ de cada localização " $i$ " para cada grupo de usuários " $u$ " pelo procedimento WLC, é possível determinar o nível de acessibilidade global pelo procedimento OWA (ver Rodrigues da Silva et al., 2008). Este último possibilita a definição de cenários através de combinações de diferentes vetores de pesos ordenados (order weights), através da Equação 2.

$$
A_{i}^{g}=A^{i} \cdot O^{T}
$$

sendo,

$A_{i}^{g}$ : índice de acessibilidade global de um local $i$;

$A^{i}$ : vetor com valores $A_{i}^{u}$ ordenados em ordem crescente (para o local i);

$O^{T}$ : vetor transporto de order weights, de dimensão igual ao número de usuários $u$.

Dentro do espaço de decisão, os seguintes cenários são considerados extremos: 1) o cenário pessimista ou conservador, onde é aplicado todo o peso do vetor de order weights ao índice com menor valor, produzindo a solução de risco mínimo; 2) o cenário ponderado, 
em que é aplicado um conjunto de order weights de valor igual para todos os índices, produzindo uma solução de risco médio; 3) o cenário otimista, onde todo o peso do vetor de order weights é aplicado ao índice com valor mais elevado, produzindo uma solução dita de alto risco.

\section{ESTUDO DE CASO E CARACTERIZAÇÃO DA ÁREA}

Neste trabalho foi realizado um estudo de caso dentro do campus de Gualtar da Universidade do Minho, em Braga, Portugal. A escolha se deu pelo fato de este campus ter sido objeto de estudo em pesquisa anterior realizada por Rodrigues (2001), que efetuou uma avaliação multicritério da acessibilidade aos seus diversos destinos-chave, do ponto de vista do pedestre. Porém, a pesquisa então realizada considerou apenas a locomoção de usuários sem deficiência aparente.

A área analisada possui cerca de 12 hectares e é dotada de infraestrutura urbana que comporta diversas Escolas e Institutos, três Complexos Pedagógicos e vários serviços, com destaque para a Cantina e a Biblioteca. Segundo Rodrigues (2001), a comunidade universitária do campus compreendia naquela ocasião aproximadamente 13100 usuários, sendo 12000 alunos, 800 docentes e 300 funcionários, sobre os quais incidiu a pesquisa de opinião referida no item 3.1. No que respeita aos destinos-chave, o campus foi dividido em 3 grupos, de acordo com a sua funcionalidade principal: pedagógico (salas de aula, laboratórios, escolas, departamentos e institutos), serviços (biblioteca, serviços acadêmicos, cantina, bancos, etc.) e acessos (entradas e estacionamentos), sendo que estes grupos encontram-se distribuídos de forma dispersa dentro do campus (Figura 4). Para o estudo de caso, de forma a simplificar a aplicação do método, mas sem comprometer a sua validação, foram apenas considerados os destinos-chave do grupo pedagógico.

Dentro da área avaliada, os deslocamentos podem ser feitos a pé, por bicicleta ou por modo motorizado, sendo que o acesso ao campus pode ser realizado por três entradas (Figura 4), com certas especificidades para alguns modos. Quanto aos modos a pé e bicicleta, pode-se usar todos os acessos. Para o modo motorizado, somente as portarias 1 e 3 permitem o tráfego de veículos. Como a topografia do campus é bastante acidentada, a ocorrência de rampas e escadarias é frequente. Algumas das rampas têm inclinações acima do recomendado pelas normas técnicas de acessibilidade, chegando-se a observar rampas com inclinações transversais de até $12,20 \%$ e outras longitudinais de até $27,90 \%$. A maioria das calçadas encontra-se em bom estado de conservação, contudo nem todas possuem largura mínima adequada por existirem obstáculos que reduzem sua faixa livre/efetiva. Em alguns pontos das vias foram implantadas faixas de travessia com guias rebaixadas nas calçadas, que proporcionam ao pedestre maior segurança e conforto quando cruzam as vias de veículos.

Na Figura 5 é possível visualizar a rede de circulação para pedestres dentro do campus que foi utilizada nesta pesquisa. Embora na prática muitos pedestres circulem pelo leito carroçável, a rede criada para análise foi definida a partir do critério de existência de calçadas, sendo utilizada a via de veículos apenas em locais onde não existem calçadas. Optou-se por utilizar sentido de fluxo na rede por existir uma grande diferença de velocidade (entre subidas e descidas) causada pela topografia. As escadarias possuem dimensões de piso e espelho dentro do recomendado por norma, entretanto algumas delas não possuem corrimãos. Outras dispõem de corrimãos inadequados (fora do recomendado por norma), apresentando risco para pessoas com deficiência visual e para idosos. Justifica-se ainda referir que em grande parte do campus praticamente não existe tratamento adequado para pessoas com deficiência visual, exceto em alguns pontos específicos.

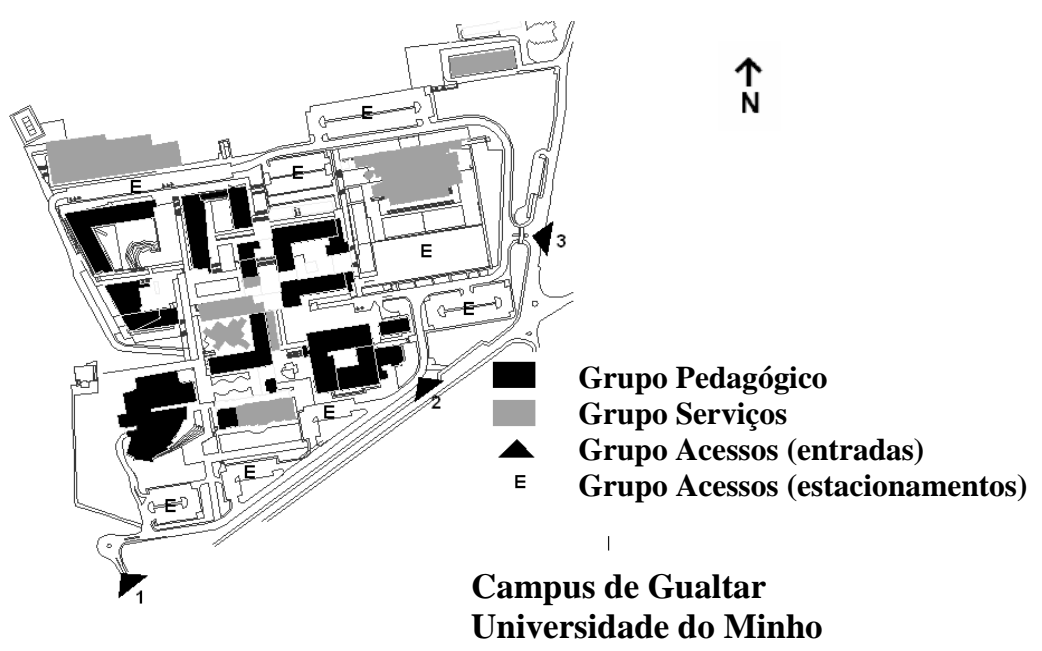

Figura 4. Distribuição dos grupos de funcionalidades dentro do campus de Gualtar 


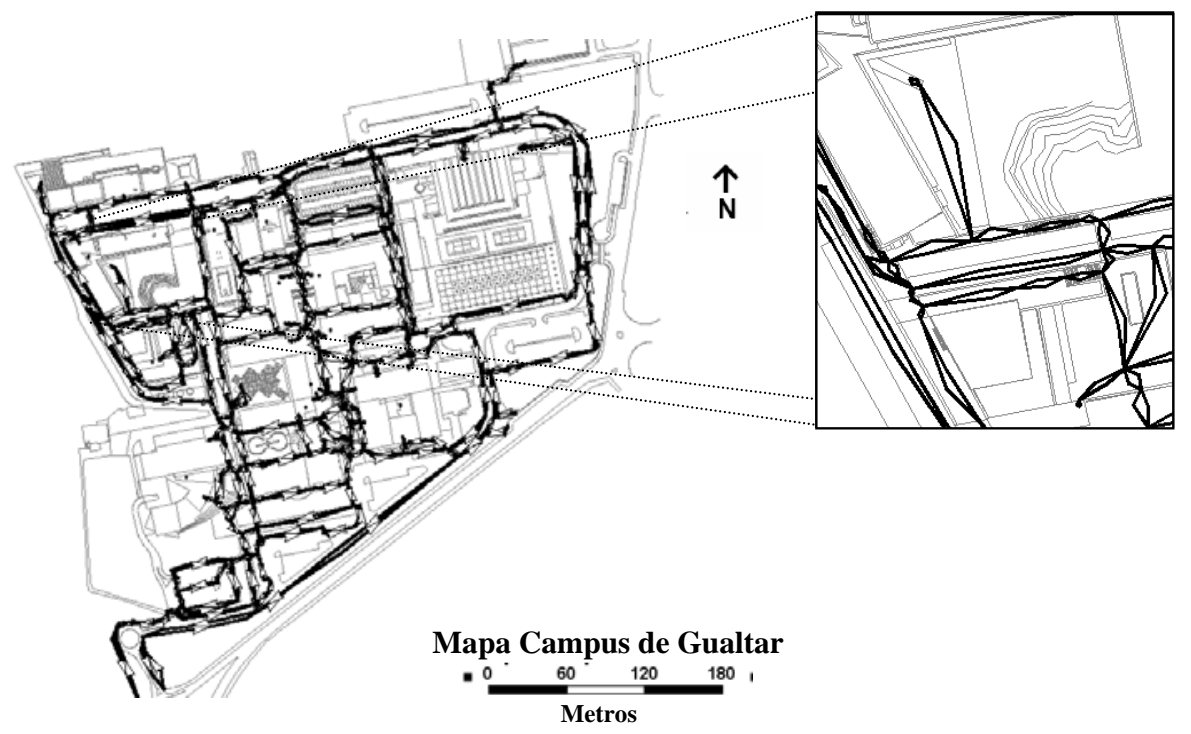

Figura 5: Mapa do Campus de Gualtar em Braga com rede de pedestres e detalhe de um trecho com identificação do sentido do fluxo

\section{APRESENTAÇÃO E ANÁLISE DOS RESULTADOS}

Através do método de avaliação multicritério e das técnicas a ele associadas, somando-se ainda a sua implementação em ambiente SIG, foi possível representar os resultados em mapas de fácil interpretação e análise. Pelo procedimento WLC de avaliação, os mapas referentes aos resultados obtidos para cada grupo de usuários apresentam os níveis de acessibilidade divididos em quatro classes normalizadas no intervalo entre zero e um. A faixa entre 0,00 e 0,25 representa o nível de menor acessibilidade e a faixa entre 0,75 e 1,00 corresponde ao nível de maior acessibilidade. Os valores máximos, as médias e os desvios padrões dos níveis de acessibilidade, partindo-se de cada origem analisada para todos os destinos-chave do setor pedagógico, são mostrados na Tabela 1, de acordo com o grupo de usuários. Portanto, a Tabela 1 permite uma breve visualização dos valores obtidos antes da normalização.

Tabela 1. Resumo dos valores do nível de acessibilidade obtidos por grupos de usuários aos destinos-chave

\begin{tabular}{lccc}
\hline & Máximo & Média & $\begin{array}{c}\text { Desvio } \\
\text { Padrão }\end{array}$ \\
\hline $\mathrm{A}_{\mathrm{i}}$ sem Def. Aparente & 0,47 & 0,25 & 0,12 \\
\hline $\mathrm{A}_{\mathrm{i}}$ Cadeirante & 0,33 & 0,14 & 0,09 \\
\hline $\mathrm{A}_{\mathrm{i}}$ Def. Visual & 0,42 & 0,21 & 0,12 \\
\hline $\mathrm{A}_{\mathrm{i}}$ idoso & 0,46 & 0,24 & 0,12 \\
\hline
\end{tabular}

Os mapas das Figuras 6 e 7, além de permitirem a visualização dos níveis de acessibilidade obtidos, também permitem identificar a localização dos destinos-chave do grupo pedagógico (grupo utilizado na análise), representados no mapa através de pontos. Assim, os resultados da aplicação do método podem ser visualizados com mais facilidade. O mapa da Figura 6a apresenta o resultado dos níveis de acessibilidade aos destinos-chave do setor pedagógico para o grupo de usuários sem deficiência aparente. Como as análises foram feitas em função dos tempos de deslocamento, os espaços com menor nível de acessibilidade $(0,00$ a 0,25$)$ indicam que tais tempos superam a média dos tempos máximos verificados para esse grupo de usuários. Os espaços com maior nível de acessibilidade (faixa que compreende os valores de 0,75 a 1,00 ), encontram-se situados na zona central do campus. Este fato é justificado por concentrarem-se nesta área grande parte dos destinos-chave avaliados, o que diminui o tempo gasto nos trechos percorridos durante a caminhada.

Para aplicação do método e simplificação da análise foram adotados fatores de redução de velocidade nos percursos que possuem rampas e escadas (item 3.3). Tanto para o grupo sem deficiência aparente quanto para o grupo de usuários com restrição de mobilidade (idosos), o fator de redução adotado corresponde a $30 \%$ da velocidade em plano horizontal, o que representa aumento de 30\% no tempo percorrido. O mapa da Figura 6b apresenta o resultado do nível de acessibilidade aos destinos-chave do setor pedagógico para o grupo de usuários com restrição de mobilidade (idosos). Como para este grupo o fator considerado foi igual ao fator usado para o grupo anterior, o mapa de acessibilidade (após normalização) do grupo de idosos (Figura 6b) assumiu valores bem aproximados do grupo de pessoas sem deficiência aparente (Figura $6 a)$.

Os resultados da acessibilidade para o grupo de pessoas com deficiência visual (cegos) podem ser vistos na Figura 7a. O nível de acessibilidade desse grupo é 
menor que o obtido para os dois grupos de usuários anteriormente mencionados (Figura 6). Este fato pode ser explicado em função dos tempos de percurso indicarem valores que superam a média dos tempos máximos atribuídos pelas pessoas com deficiência visual. Foram adotadas impedâncias em rampas, escadas e travessias de ruas sem sinalização adequada para este grupo, que correspondeu a uma velocidade 20\% menor que em trechos de calçada em plano horizontal. $\mathrm{Na}$ análise do mapa de acessibilidade para o grupo de cadeirantes (Figura 7b), pode ser observada uma redução significativa do tamanho da área de maior acessibilidade $(0,75$ a 1,00$)$. O nível mais alto ocorreu nas áreas mais planas do entorno dos prédios do setor pedagógico, uma vez que os cadeirantes não conseguem circular nos trechos em escada e em travessias de ruas com guias sem rebaixamento (item 3.3).

Em todos os mapas, os maiores níveis de acessibilidade foram apresentados em forma de bolsões que podem ser justificados pelo fato das doze edificações, pertencentes ao setor pedagógico (pontos demarcados nos mapas), estarem agrupadas nessas áreas. Vale lembrar que, para o estudo de caso, foram apenas consideradas como destinos-chave as edificações que fazem parte do grupo pedagógico, de forma a simplificar a aplicação do método. $\mathrm{Na}$ análise, percebe-se que praticamente $50 \%$ destas edificações possuem o maior nível de acessibilidade para três grupos de usuários (sem deficiência, com restrição de mobilidade e com deficiência visual) sendo que apenas duas edificações apresentaram o mesmo nível para o grupo de usuários com deficiência física (cadeirantes).

Segundo o procedimento OWA (que combina a informação proveniente dos resultados obtidos para cada grupo de usuários, Figuras 6 e 7) foram desenvolvidos seis cenários diferentes, os quais foram elaborados a partir de combinações dentro do espaço estratégico de tomada de decisão (Rodrigues da Silva et al., 2008). Para tanto, foram definidos os pontos de con-

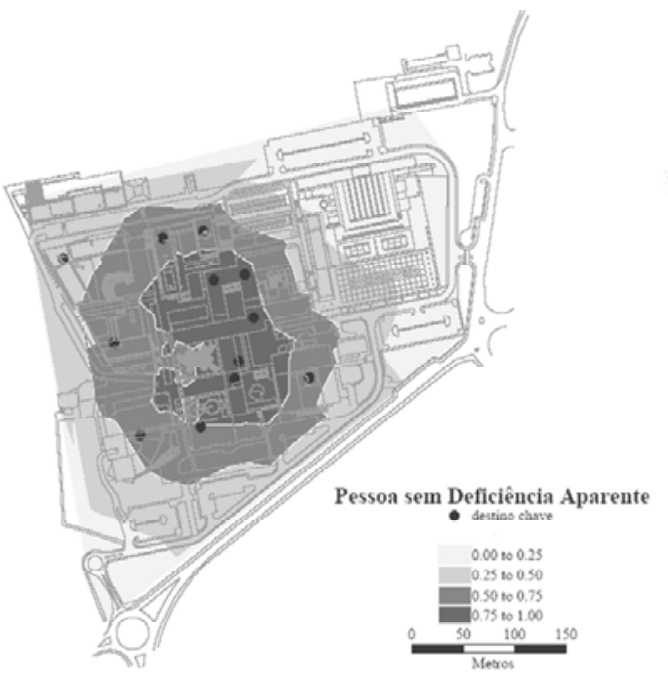

(a)

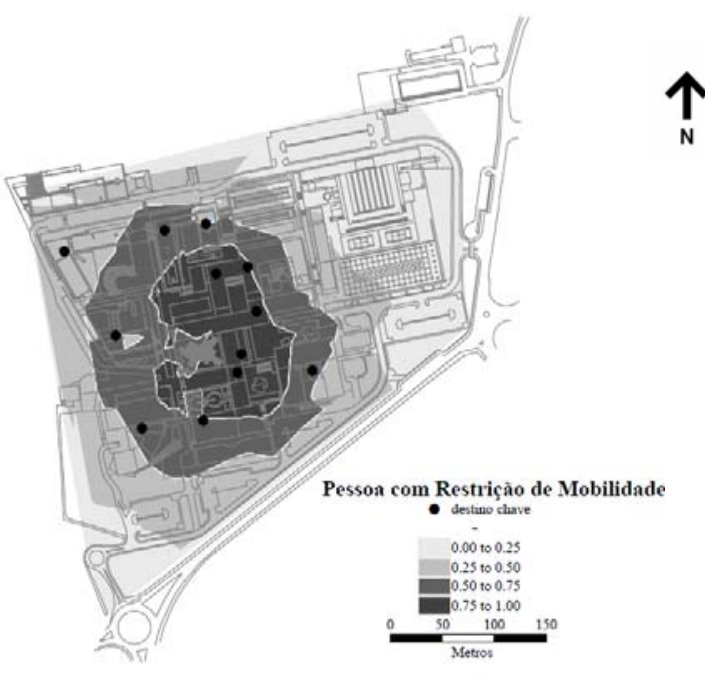

(b)

Figura 6. Níveis de acessibilidade aos destinos-chave do setor pedagógico (a) para o grupo de usuários sem deficiência aparente e (b) para o grupo de usuários com restrição de mobilidade - idosos

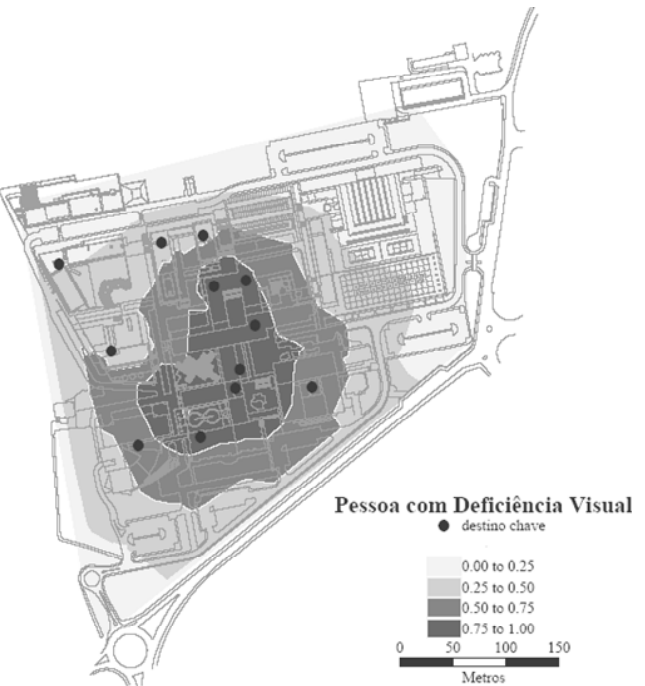

(a)

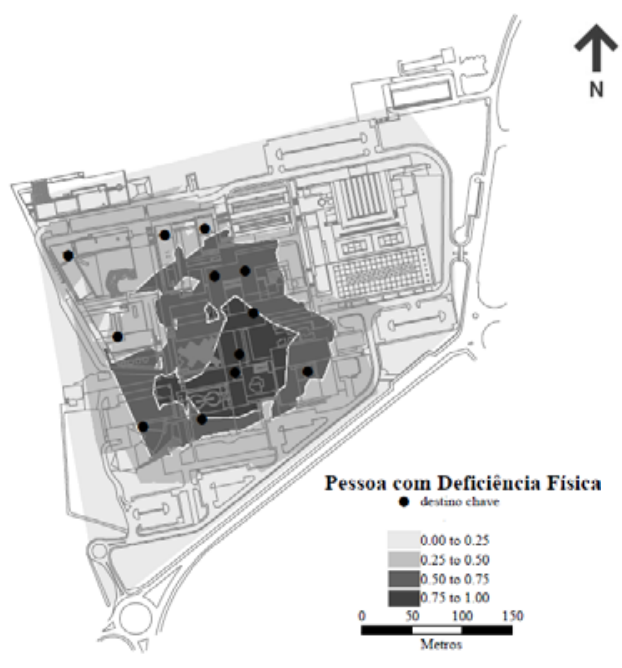

(b)

Figura 7. Níveis de acessibilidade de pessoas (a) com deficiência visual e (b) de cadeirantes 
trole (ou decisão) A, B, C, D, E e F, cujos respectivos vetores order weights são apresentados na Tabela 2. Os referidos pontos de decisão são mostrados na Figura 8.

Os mapas referentes aos resultados obtidos para estes seis cenários também apresentam os níveis de acessibilidade divididos em quatro classes normalizadas no intervalo entre zero e um. A faixa entre 0,00 e 0,25 representa o nível de menor acessibilidade e a faixa entre 0,75 e 1,00, o nível de maior acessibilidade. Os valores máximos, as médias e os desvios padrões das acessibilidades, em cada cenário (depois de aplicados os vetores order weights) são mostrados na Tabela 3. Portanto, a Tabela 3 permite uma breve visualização dos valores obtidos antes da normalização.

Os mapas da Figura 9 representam os três cenários obtidos a partir dos pontos A, B e C (pontos extremos do triângulo que determina o espaço estratégico de tomada de decisão). No cenário A (Figura 9a) foram aplicados pesos (OW) de valores iguais aos fatores, o que produziu uma solução de risco médio e trade-off máximo, ou seja, um cenário intermediário ou balanceado com compensação total entre os fatores considerados. No cenário B (Figura 9b) todo o peso foi aplicado ao fator de mais alto valor, o que produziu uma situação de risco elevado (denominada otimista) e que se aproxima do cenário obtido para os usuários sem deficiência aparente. No cenário C (Figura 9c) todo o peso foi aplicado ao fator de mais baixo valor, produzindo assim, uma solução de risco mínimo (denominada de pessimista) e que se aproxima do cenário produzido para os cadeirantes.

Os mapas da Figura 10 representam os três cenários obtidos a partir dos pontos D, E e F (pontos intermediários dentro do triângulo que determina o espaço estratégico de tomada de decisão). No cenário D (Figura 10a), pesos de valores diferentes foram aplicados aos fatores, de forma a gerar uma condição de risco médio e trade-off médio. Logo, o cenário obtido representa uma situação intermediária entre os três cenários extremos (A, B e C), ou seja, os cenários ponderado, pessimista e otimista. No cenário E (Figura 10b) também foram aplicados pesos de valores diferentes aos fatores, entretanto enfatizou-se o fator de maior escore. Isto produziu uma situação de risco elevado (avaliação próxima da otimista) e que se aproxima do cenário produzido para os usuários sem deficiência aparente, entretanto, possui trade-off médio. Por último, no cenário F (Figura 10c), pesos de valores diferentes foram aplicados aos fatores de forma a enfatizar, desta vez, o fator de menor escore. Isso produziu uma situação de risco baixo (avaliação próxima da pessimista) e que se aproxima do cenário gerado para os cadeirantes, entretanto, possui trade-off médio.

Tabela 2: ANDness e Trade-off para os seis pontos de decisão utilizados

\begin{tabular}{ccccccc}
\hline \multirow{2}{*}{ Pontos de decisão } & \multicolumn{3}{c}{ Order Weigths } & & $\begin{array}{c}\text { ANDness } \\
\text { nível de risco }\end{array}$ & $\begin{array}{c}\text { Trade-off } \\
\text { nível de compensação }\end{array}$ \\
\hline A & 0,250 & 0,250 & 0,250 & 0,250 & 0,50 & 1,00 \\
\hline B & 0,000 & 0,000 & 0,000 & 1,000 & 0,00 & 0,00 \\
\hline C & 1,000 & 0,000 & 0,000 & 0,000 & 1,00 & 0,00 \\
\hline D & 0,036 & 0,464 & 0,464 & 0,036 & 0,50 & 0,50 \\
\hline E & 0,126 & 0,126 & 0,126 & 0,622 & 0,25 & 0,50 \\
\hline F & 0,622 & 0,126 & 0,126 & 0,126 & 0,75 & 0,50 \\
\hline
\end{tabular}

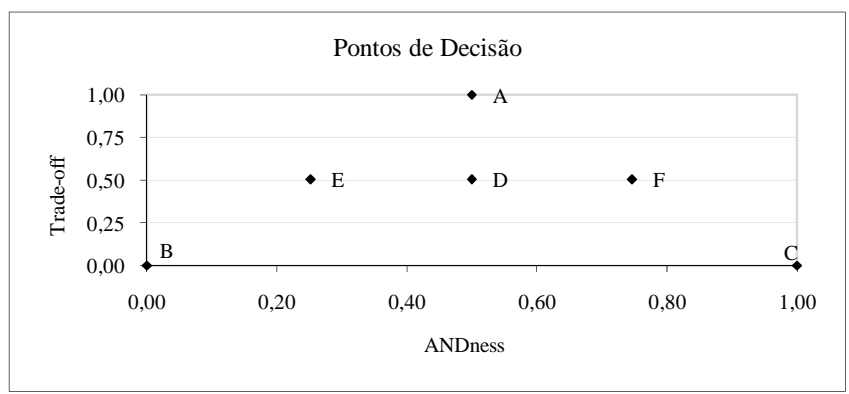

Figura 8. Pontos de decisão dentro do espaço estratégico utilizado

Tabela 3. Resumo dos valores de acessibilidade obtidos para os seis cenários

\begin{tabular}{lcccccc}
\hline & $\boldsymbol{A}$ & $\boldsymbol{B}$ & $\boldsymbol{C}$ & $\boldsymbol{D}$ & $\boldsymbol{E}$ & $\boldsymbol{F}$ \\
\hline Máximo & 0,41 & 0,47 & 0,33 & 0,43 & 0,44 & 0,37 \\
\hline Média & 0,21 & 0,25 & 0,13 & 0,22 & 0,23 & 0,17 \\
\hline Desvio padrão & 0,11 & 0,12 & 0,09 & 0,11 & 0,11 & 0,10 \\
\hline
\end{tabular}




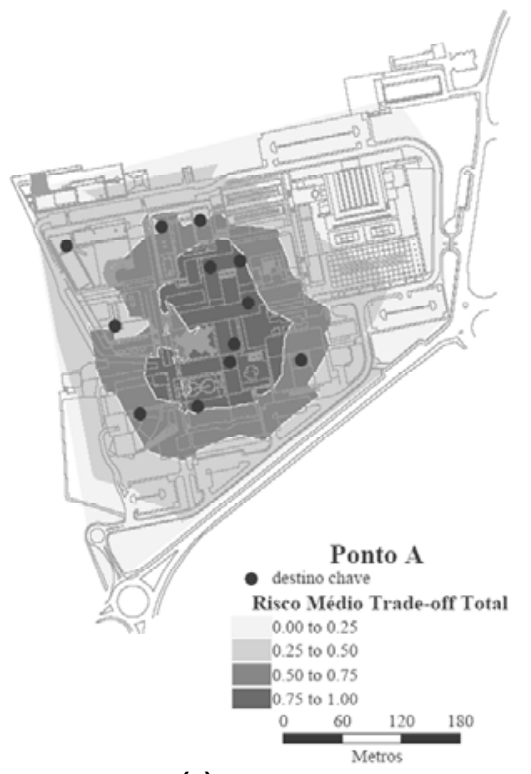

(a)

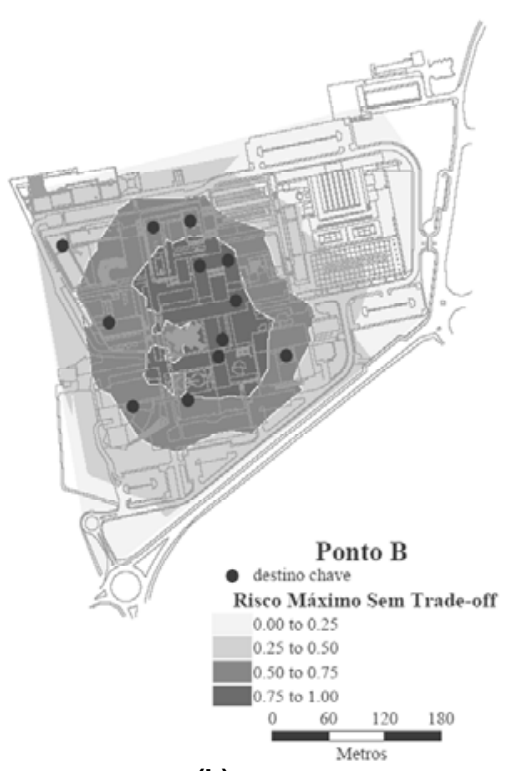

(b)

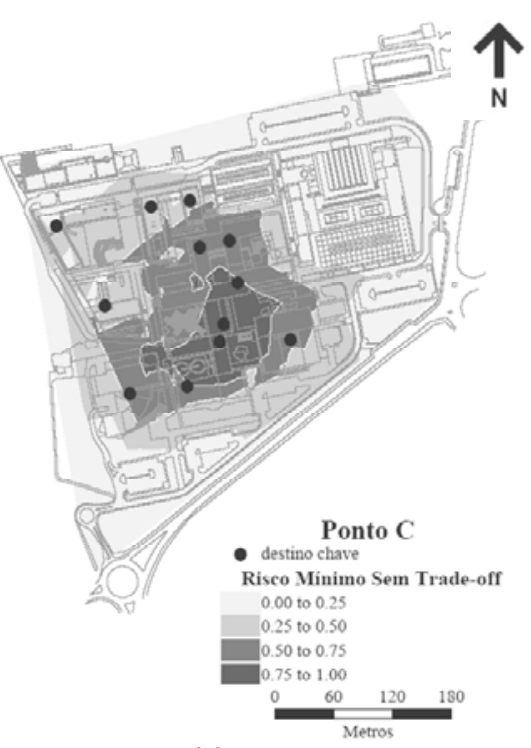

(c)

Figura 9. Níveis de acessibilidade para o cenário balanceado (a), cenário otimista (b) e cenário pessimista (c)

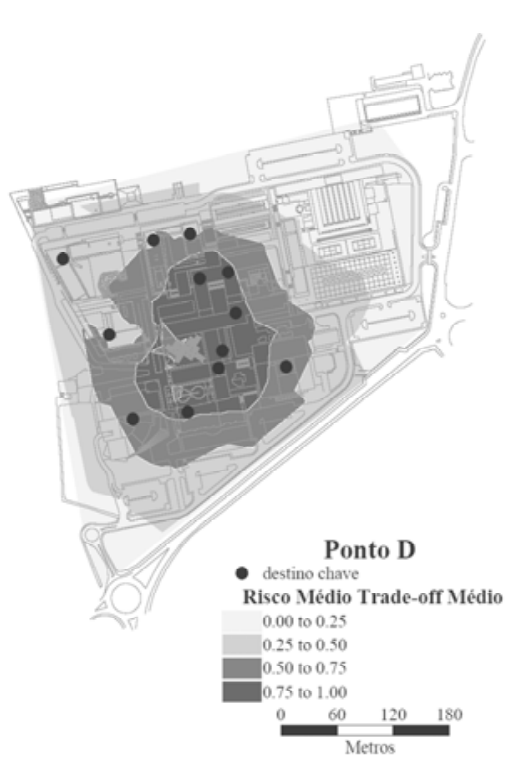

(a)

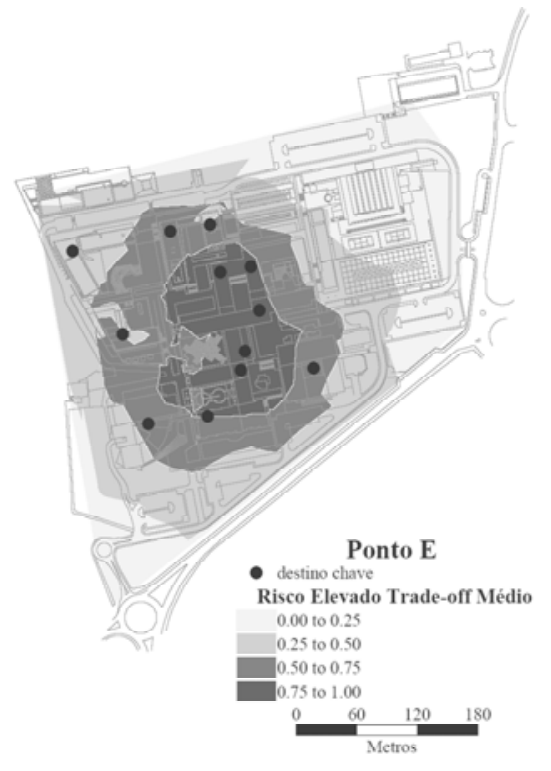

(b)

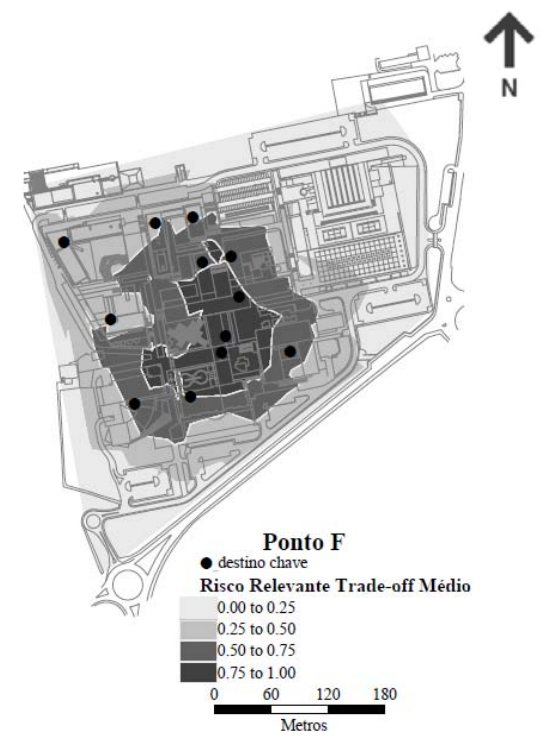

(c)

Figura 10. Níveis de acessibilidade de cenários intermediários relativo ao ponto de decisão D (a), ponto E (b) e ponto F (c)

\section{CONCLUSÕES}

A aplicação do método de avaliação multicritério em ambiente SIG, com o objetivo de verificar as condições de mobilidade em calçadas e travessias de quatro grupos de usuários selecionados, conduziu a algumas conclusões importantes. A princípio, o estudo de caso em um campus universitário foi fundamental para consolidar e definir os critérios a serem utilizados na avaliação multicritério. Os principais valores de referência para a análise foram: os tempos de percurso para cada grupo de usuários e a importância dos destinos selecionados. A aplicação no sítio escolhido valida o método e sugere a sua ampliação para avaliar o ambiente urbano em geral.
A representação dos resultados em mapas temáticos (mapas de base) possibilitou identificar os níveis de acessibilidade de cada grupo de usuários dentro da área estudada. Confirmou-se assim que os níveis de acessibilidade variam em função do grupo de usuários, sendo em geral melhor para os grupos de pessoas sem deficiência aparente e para as pessoas com restrição leve de mobilidade (e.g., idosos, gestantes, obesos). Os grupos com piores níveis de acessibilidade foram os usuários com restrições na locomoção resultantes de deficiência visual ou deficiência física, no caso, cadeirantes. Embora os mapas que explicitam a distribuição espacial dos níveis de acessibilidade apresentem resultados diferenciados, como a pesquisa foi limitada somente à análise da acessibilidade ao setor pedagógico do campus, ficou evidente que a disposi- 
ção das edificações influenciou o nível de acessibilidade obtido. Esta disposição, aliada à topografia acidentada dentro do campus, chega a comprometer a mobilidade de muitos usuários, sobretudo os cadeirantes.

Por outro lado, fazendo uso de um cenário dito pessimista, obtido através da combinação OWA, foi possível representar os resultados em mapas com cenários de avaliação que permitem ao planejador atribuir uma importância maior às categorias de usuários mais prejudicados e que são frequentemente mal atendidas.

Em síntese, as conclusões do trabalho sugerem que o método é adequado e promissor, pois possibilita obter indicadores para a caracterização das condições de mobilidade em espaços urbanos. Por outro lado, a utilização de ferramentas de simulação espacial pode permitir criar cenários com base em propostas de melhorias a serem introduzidas na rede de circulação dos pedestres. Estes cenários podem ajudar o planejador a escolher as ações que conduzem a resultados mais eficientes, comparando e identificando os ganhos relativamente ao cenário de partida. Desse modo, é possível pré-avaliar a adoção de ações para reduzir as deficiências e/ou ineficiências dos espaços de circulação urbana. O modelo ainda permite a introdução de análises mais aprofundadas, que podem vir a sugerir inclusive a necessidade da incorporação de um maior número de critérios que servirão para refinar os resultados.

\section{AGRADECIMENTOS}

Os autores agradecem à CAPES (Coordenação de Aperfeiçoamento de Pessoal de Nível Superior) pelo apoio financeiro no âmbito do Programa de Doutorado no País com Estágio no Exterior (PDEE) durante a realização da pesquisa que originou o presente trabalho.

\section{REFERÊNCIAS BIBLIOGRÁFICAS}

Aguiar, F. de O.; A. N. Rodrigues da Silva; R. A. R. Ramos e V. N. Yuassa (2008) Avaliação da Mobilidade em Espaços Urbanos com Deficiências para Pessoas com Dificuldade de Locomoção. III Congresso Luso Brasileiro para o Planejamento Urbano, Regional, Integrado e Sustentável - PLURIS 2008. Santos, SP, Brasil.

Almeida, A. L. de J. (2006) A Pessoa com Deficiência em Portugal e Brasil: Desafios para Ações em Saúde. HYGEIA, Revista Brasileira de Geografia Médica e da Saúde. v. 2, n. 3, dez. 2006. Disponível em: <http://www.hygeia.ig.ufu.br/>. Acesso em: 3 mar. 2009.

Church, R. L e J. R. Marston (2003) Measuring Accessibility for People with a Disability. Geographical Analysis, v. 35, n. 1, p. 81-96.

Cohen, R. (2006) Cidade, Corpo e Deficiencia: Percursos e Discursos Possíveis na Experiência Urbana. Tese de Doutorado. Rio de Janeiro: EICOS/IP/UFRJ, 213 p.

Duarte, C. R. e R. Cohen (2004). Arquitetura, Espaço, Acesso e Afeto: A Construção de uma Experiência Afetiva por Pessoas com Dificuldade de Locomoção. Seminário Internacional Sociedade Inclusiva. PUC-Minas. Disponível em: $<$ http://www.bengalalegal.com/afetoelugar.php>. Acesso em: 15 set. 2007.

Feijó, A. R A. (2002). Direitos Humanos e Proteção Jurídica da Pessoa Portadora de Deficiência: Normas Constitucionais de Acesso e Efetivação da Cidadania à Luz da Constituição Federal de 1988. Brasília: Ministério da Justiça, Secretaria de Estado dos Direitos Humanos, 184 p.
Little, J. (1995) What has ADA Really Done? Accent on Living, v. 39, n. 4, p.28-30.

INE (2002) Instituto Nacional de Estatística - Portugal. Censos 2001: Análise de População com Deficiência, Resultados Provisórios. Lisboa, INE, 4 de Fevereiro de 2002. Informação à comunicação social. Disponível em: <http://www.ine.pt.> Acesso em: $8 \mathrm{fev}$. 2009.

Martins, J. V. (2008) Projectar e Construir sem Barreiras - DL 163/06 de 8 de Agosto - Anotado e Comentado. Ed. DisLivro. Lisboa. Portugal. $194 \mathrm{p}$.

Mendes, J. F. G. (2000) Decision Strategy Spectrum for the Evaluation of Quality of Life in Cities. In: Proceedings of the International Conference on Quality of Life in Cities - ICQOLC 2000, Cingapura.

Mohammadian, A. e S. Bekhor (2008) Travel Behavior of Special Population Groups. Transportation, v. 35, n. 5, p. 579-583.

ONU - Organização das Nações Unidas. (2007) Convention on the Rights of Persons with Disabilities and Optional Protocol. Disponível em: <http://www.un.org/disabilities/documents/convention/ convoptprot-e.pdf.> Acesso em: 20 ago 2009.

Páez A.: R. J. Mercado; S. Farber; C. Morency e M. Roorda (2009) Mobility and Social Exclusion in Canadian Communities: An Empirical Investigation of Opportunity Access and Deprivation from the Perspective of Vulnerable Groups, Report to Policy Research Directorate, Strategic Policy and Research, Human Resources and Social Development Canada.

Portugal (2006). " $1^{\circ}$ Plano de Acção para a Integração das Pessoas com Deficiências ou Incapacidade”, Ministério do Trabalho e da Solidariedade Social. Disponível em: <http://www.inr.pt/ content/1/26/paipdi/ > Acesso em: 10 abr. 2009.

Prado, A. R. de A. (1997). Ambientes Acessíveis. Artigo publicado no documento sobre o Primeiro Seminário Nacional "A Pessoa Portadora de Deficiência no Mundo do Trabalho", CORDE. Disponível em: <http://www.entreamigos.com.br/>. Acesso em: 20 jun. 2008.

Rodrigues, D. S. (2001) Avaliação Multicritério da Acessibilidade em Ambiente SIG. Dissertação de Mestrado. Universidade do Minho, Braga, Portugal. 144 p.

Rodrigues da Silva, A. N.; R. A. R. Ramos; L. C. L. de Souza; D. S. Rodrigues e J. F. G. Mendes (2008) SIG: Uma Plataforma para Introdução de Técnicas Emergentes no Planejamento Urbano, Regional e de Transportes. São Carlos, SP: EdUFSCar. 227 p.

Schmöcker, J. D.; M. A. Quddus; R. B. Noland e M. G. H. Bell (2008) Mode Choice of Older and Disabled People: a Case Study of Shopping Trips in London. Journal of Transport Geography, v. 16 , n. 4, p. 257-267.

Schmöcker, J. D. (2009) Access, Aging, and Impairments Part A: Impairments and Behavioral Responses. Journal of Transport and Land Use, v. 2, n. 1, p. 1-2. 